\title{
APLICABILIDADE DA CLASSIFICAÇÃO DOS RESULTADOS DE ENFERMAGEM EM PACIENTES COM DÉFICIT NO AUTOCUIDADO: banho/ higiene ${ }^{a}$
}

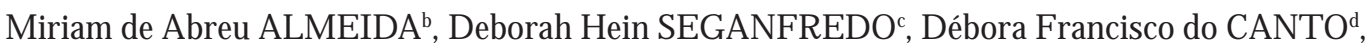 \\ Luciana Nabinger MENNA BARRET Oe
}

\section{RESUMO}

O objetivo da pesquisa é verificar a aplicabilidade da Classificação dos Resultados de Enfermagem (NOC) em pacientes ortopédicos hospitalizados com o diagnóstico de enfermagem D éficit no Autocuidado: banho/ higiene. E studo quantitativo, descritivo, desenvolvido em hospital universitário com pacientes submetidos à artroplastia total de quadril em 2008. 0 instrumento de coleta de dados continha 24 indicadores dos resultados da NOC Autocuidado: Atividades de Vida Diária, Autocuidado: Banho, A utocuidado: Higiene, Autocuidado: Higiene Oral e uma escala Likert onde $1=$ dependente; $2=$ não participa; 3 = necessita de pessoa auxiliar; 4 = independente com mecanismo auxiliar; 5 = completamente independente. Foi observado o banho de 25 pacientes no primeiro e terceiro dia de pós-operatório. Os aspectos éticos foram respeitados. E $\mathrm{m} 21$ indicadores houve variação estatisticamente significativa entre as médias de pontuação do primeiro para o segundo banho. As escalas da Classificação NOC apresentaram consistência interna, mostrando-se confiáveis para mensurar a evolução dos pacientes durante a implementação dos cuidados de enfermagem.

D escritores: Processos de enfermagem. Classificação. Avaliação de resultados (cuidados de saúde). E nfer magem ortopédica.

\section{RESUMEN}

E I objetivo de la investigación es verificar la a plicabilidad de la Clasificación de R esultados deE nfermería (N OC) en pacientes ortopédicos hospitalizados con diagnóstico de enfer mería D éficit en el A utocuidado: baño/ higiene. E studio cuantitativo, descriptivo, desarrollado en hospital universitario con pacientes someti dos a ar toplastía total de cadera en 2008. El instrumento de recolección de datos contenía 24 indicador es de resultados de la N OC A utocuidado: A ctividades de Vida D iaria, A utocuidado: Baño, A utocui dado: H igiene, A utocuidado H igiene 0 ral y una escala de $L$ ikert en la que 1 = dependiente; 2 = no partici pa; 3 = necesita una persona auxiliar; 4 = independiente con mecanismo auxiliar; 5 = completamente independiente Se observó el baño de 25 paci entes en el primer y tercer días de postoperatorio. L oS aspectos éticos fueron respetados. E n 21 indicadores hubo variación estadísticamente significativa entre las medias de puntuación del primero para el segundo baño. L as escal as de la Clasificación N OC presentaron consistencia interna, mostrándose confiables para mensurar la evolución de los pacientes durante la implementación de los cuidados de enfermería.

D escriptores: P rocesos de enfermería. Clasificación. E valuación de resultado (atención de salud). E nfermería ortopédica.

T ítulo: A plicabilidad de la clasificación de resultados de enfermería en pacientes con déficit en el autocuidado: baño/ higiene

\section{ABST RACT}

T he pur pose of this research is to explore the $\mathrm{N}$ ursing 0 utcomes Classification (N OC) applicability on hospitalized or thopedic patients diagnosed with Self-careD eficit: bathing/ hygiene. This is a quantitative, descriptivestudy devel oped in a university hospital with patients submitted to total hip replacement in 2008. The data collection instrument was composed of $24 \mathrm{~N} \mathrm{OC} \mathrm{Self-careoutcome} \mathrm{indicators:} \mathrm{Self-}$ care: Activities of $\mathrm{D}$ aily L iving; Self-care: Bathing; S elf-care: $\mathrm{H}$ ygiene; $\mathrm{Self}$ - care: $O$ ral $\mathrm{H}$ ygiene; and a L ikert scalein which $1=$ dependent; $2=$ does not participate; $3=$ requires an assistant; $4=$ independent but requires an assistant mechanism; $5=$ completely independent. 25 patients bathing on first and third post-operative day w ere observed. E thical aspects were respected. In 21 cases there was significant statistical variation betw een average scores from first to second bath. N OC classification scales presented internal consi stency, proving their reliability to measure the patient evolution during nursing care implementation.

Descriptors: N ursing process. Outcome assessment (health care). Classification. O rthopedic nursing.

T itle: N ursing outcomes classification applicability on patients with self-care deficit: bathing/ hygiene.

\footnotetext{
a Segunda etapa do projeto "Resultados de Enfermagem no Cuidado Corporal em Pacientes Ortopédicos - Viabilidade da N ursing Outcomes Classification em um Hospital U niversitário".

b D outora em E ducação, Professor Adjunto da Escola de Enfermagem da Universidade Federal do Rio Grande do Sul (UFRGS), Porto Alegre, Rio Grande do Sul, Brasil.

c M estre em Enfermagem, Residente em Atenção ao Paciente Crítico do Grupo Hospitalar Conceição, Porto Alegre, Rio Grande do Sul, Brasil.

d Enfermeira, M estranda do Programa de Pós-G raduação em Enfermagem da UFRGS, Porto Alegre, Rio Grande do Sul, Brasil.

e A cadêmica da E scola de Enfermagem da U FRGS, Bolsista do Programa Institucional de Bolsas de Iniciação Científica (PIBIC/U F RGS/ CN Pq), Porto Alegre, Rio G rande do Sul, Brasil.
} 


\section{INT RODUÇÃO}

Os sistemas de classificação consistem em terminologias padronizadas empregadas nas diferentes etapas do processo de enfermagem (PE), metodologia utilizada na organização do conhecimento e do cuidado individualizado ao paciente. Este método pode ser entendido como uma atividade intelectual deliberada que auxilia o enfer meiro na tomada de decisões, cujo foco reside na obtenção dos resultados esperados.

O H ospital deClínicas de Porto A legre(HCPA), local do estudo, vem desenvolvendo o processo de enfermagem na prática clínica em cinco etapas: anamnese e exame físico, diagnóstico de enfermagem, prescrição dos cuidados, implementação e evolução/ avaliação(1). A etapa do diagnóstico de enfer magem ( $D E$ ) foi inserida e informatizada no ano de 2000 utilizando o vocabulário da T axonomia I da N orth American N ursing Diagnosis A ssociation - International (NANDA-I), eposteriormente da Taxonomia II, conjugado ao referencial de Wanda $\mathrm{H}$ orta que se fundamenta na T eoria das Necessidades H umanas Básicas da M aslow ${ }^{(2,3)}$.

A partir de 2005 têm sido introduzidas intervenções/ atividades da $\mathrm{N}$ ursing Interventions Classification (NIC)(4) no Sistema I nformatizado de Prescrição de Enfermagem. Contudo, na avaliação dos resultados de enfermagem não é empregada terminologia padronizada, sendo uma das metas do G rupo de Enfermagem estudar a N ursing 0 utcomes Classification ( $\mathrm{NOC})^{(5)}$ e investigar sua viabilidade para futura implantação.

A NOC está sendo desenvolvida por pesquisadores da U niversidade de I owa, assim como a N IC, e podem ser usadas em conjunto com a NANDA-I. A construção de uma classificação direcionada à avaliação da eficácia das intervenções de enfermagem teve início em 1991 ea estruturação deste sistema vem sendo realizada por pesquisadores experientes e revisada com ênfase em testes clínicos de campo, quanto à confiabilidade, validade, sensibilidade, especificidade e utilidade das medidas dos resultados ${ }^{(5)}$.

A primeira publicação da N OC, de 1997, continha 190 resultados e a segunda, datada de 2000, continha 260 resultados. A terceira edição da N OC, traduzida para o português no ano de 2008, é composta de 330 resultados agrupados em 31 classes e sete domínios e não apresentou modificações no capítulo que sugere as ligações entre as classifica- ções NANDA-I e NOC para o diagnóstico D éficit no Auto-Cuidado: banho/ higiene. Os sete domínios são: (1) Saúde F uncional; (2) Saúde P sicológica; (3) Saúde Psicossocial; (4) Conhecimento e Comportamento Saudável; (5) Saúde Percebida; (6) Saúde Familiar; e (7) Saúde Comunitária. Cada resultado pode ser encontrado em uma classe apenas e possui um único código numérico visando facilitar a sua inserção em um sistema infor matizado. A quarta edição ainda sem tradução para o português, contém 385 resultados nas mesmas classes e domínios anteriores ${ }^{(5)}$.

A NOC compreende os resultados que descrevem o estado, comportamentos, reações e sentimentos do paciente em resposta ao cuidado prestado. Cada resultado possui um rótulo ou nome, uma definição e uma lista de indicadores que descrevem o cliente, o cuidador ou a família em uma escala tipo L ikert de cinco pontos que vai do estado menos desejável ao mais desejável. As várias escalas desenvolvidas facilitam a identificação de alterações no estado do paciente por meio de diferentes pontuações ao longo do tempo. Possibilita, portanto, monitorar a mel hora, a piora ou a estagnação do estado do paciente durante um período de cuidado ou através de diferentes setores de atendimento(5).

0 presente estudo consiste na segunda etapa do projeto de pesquisa "R esultados de enfer magem no cuidado corporal em pacientes ortopédicos viabilidade da $\mathrm{N}$ ursing Outcomes Classification em um hospital universitário". Na primeira etapa foram validados por enfermeiras peritas os indicadores de quatro resultados de enfer magem da segunda edição da $N O C^{(6)}$ para pacientes com o DE D éficit no Autocuidado: banho/ higiene. Consideraram-se peritas as enfer meir as que atender am aos seguintes critérios: trabalhar em unidades de internação que atendem pacientes adultos submetidos à Artroplastia Total de Quadril (AT Q) no HCPA; ter experiência profissional mínima de dois anos; ter experiência com pacientes or topédicos de, no mínimo, um ano e utilizar o DE anteriormente mencionado com estes pacientes. E m estudo anterior verificou-se que este DE estava presente em $90 \%$ de pacientes com as mesmas características( ${ }^{(7)}$.

Déficit no Autocuidado para banho/ higiene passou a ser enunciado como $D$ éficit no autocuidado para banho, permanecendo inalterada sua definição como "capacidade prejudicada de realizar ou completar as atividades de banho/higiene por si 
mesmo"(8). Para este DE são sugeridos os seguintes resultados da N OC: A utocuidado: Banho, Autocuidado: Higiene, Autocuidado: A tividades de Vida Diária (AVD); A utocuidado: Higiene Oral e Autocuidado: Ostomia. Considerando-se que as vinculações entre as classificações não são prescritivas, optou-se por utilizar os quatro primeiros resultados apresentados nas ligações entre NANDA-I, $\mathrm{NOC}$ e N IC ${ }^{(9)}$. A penas o resultado Autocuidado: Ostomia não foi incluído por tratar-se de pacientes ortopédicos ${ }^{(4)}$. Dos 34 indicadores sugeridos para os quatro Resultados de E nfermagem, 24 foram validados pelas peritas, considerando-se a média aritmética ponderada $\geq 0,6$. Assim sendo, estes 24 indicadores farão parte deste estudo.

A escolha por estudar pacientes com problemas ortopédicos, especificamente aqueles submetidos à AT Q decorre da demanda de cuidados sob responsabilidade da E nfermagem. Este é um procedimento cirúrgico amplamente empregado no tratamento de afecções da articulação coxofemoral, sejam el as degenerativas, inflamatórias ou traumáticas $^{(10)}$

Pacientes submetidos à AT Q tornam-se dependentes da equipe de enfermagem no pós-operatório imediato, devido à limitação para mobilizar-se e à restrição ao leito, visto que não podem apoiar no chão nem realizar exercício de adução com o membro operado pelo risco de luxação da prótese. N ecessitam, portanto, de assistência para realizar as atividades de higiene pessoal, como 0 banho, normalmente realizado no leito ${ }^{(11)}$.

Espera-se, com essa investigação, contribuir com os estudos sobre a N OC e na futura implantação no P rocesso de E nfer magem informatizado do HCPA. A importância de trabal har com uma classificação que mensure resultados é per mitir a identificação do impacto, da qualidade, da efetividadee do custo das intervenções de enfermagem no cuidado ao paciente.

Assim sendo, o objetivo do estudo é verificar a aplicabilidade da N OC em pacientes com problemas ortopédicos hospitalizados com o diagnóstico de enfer magem D éficit no Autocuidado: banho/ higiene.

\section{MÉTODO}

Trata-se de um estudo contemporâneo, descritivo, de caráter quantitativo ${ }^{(12)}$, desenvolvido no HCPA, que é integ rante da R ede de H ospitais U ni- ver sitários do M inistério da E ducação e vinculado academicamente à U niversidade Federal do Rio Grande do Sul (UFRGS).

A população do estudo compreendeu os pacientes adultos com problemas ortopédicos submetidos à AT $Q$, hospitalizados em U nidades de Inter nação do Sistema Ú nico de Saúde (SUS) ou de Convênios no HCPA. Como critérios de inclusão da amostra considerou-se ter sido submetido à cirurgia ortopédica com um período de internação pós-operatória superior a 48 horas; concordar em participar da pesquisa assinando Termo de Consentimento Livre e Esclarecido. Como critério de exclusão considerou-se apresentar intercorrência no pós-operatório, período da coleta de dados.

Para coleta de dados elaborou-se um instrumento contendo oito colunas: primeira col una composta dos 24 indicadores da NOC validados em estudo anterior; segunda coluna contendo definições operacionais elaboradas pelas pesquisadoras para cada indicador; da terceira à sétima coluna, composta de escala L ikert onde 1 = dependente; 2 = não participa; 3 = necessita de pessoa auxiliar; 4 = independente com mecanismo auxiliar; 5 = completamente independente) ${ }^{(5)}$; oitava coluna destinada a observações dos pesquisadores durante 0 acompanhamento do banho. N esta coluna, quando se identificava que um indicador "não se aplicava" à situação do paciente avaliado, era registrada a situação justificando-a.

0 resultado Autocuidado: Banho tem como definição a "capacidade para limpar o próprio corpo"(6) e Autocuidado:H igiene é a "capacidade para manter a própria higiene"(6). A definição do resultado Autocuidado: Higiene Oral é a "capacidade para cuidar da própria boca e dentes"(6), enquanto o resultado Autocuidado: A tividades de Vida D iária é definido como a "capacidade para desempenhar as tarefas físicas e atividades de cuidados pessoais mais básicas"(6). D essa forma, 0 instrumento de validação constou de todos os indicadores contidos nos quatro resultados selecionados.

A coleta de dados foi realizada em dois momentos distintos por bolsistas do grupo de pesquisa, previamente treinadas. A primeira mensuração ocorreu no primeiro dia de pós-operatório e a segunda 48 horas após. Realizou-se estudo piloto visando conhecer a aplicação do instrumento e a distribuição das variáveis tempo do banho e indicadores da NOC para posterior cálculo do tama- 
nho da amostra. Para um poder de $99 \%$, a amostra constituiu-se de 25 pacientes.

0 procedimento ocorreu após a aprovação do projeto pela Comissão de Pesquisa da Escola de E nfermagem da U FRGS e do Comitê de É tica em Saúde do G rupo de Pesquisa e Pós-G raduação do HCPA, inscrito sob o protocolo $\mathrm{n}-06-230$, e a obtenção de assinatura do T er mo de Consentimento Livre e Esclarecido pelos pacientes ou familiares responsáveis, respeitando todos os aspectos éticos pertinentes à pesquisa, conforme determina a Resolução no 196/ 96 do Conselho N acional de Saú$\mathrm{de}^{(13)}$.

Para car acterização descritiva da amostra são apresentadas as variáveis qualitativas em número e freqüência, e as variáveis quantitativas simétricas em média e desvio padrão. Para análise dos dados calculou-se alfa de Crombach a fim de avaliar a consistência interna das escal as para cada um dos resultados de enfermagem. Para comparação da evolução do paciente entre a primeira e a segunda observação do banho dos pacientes utilizou-se 0 Teste t de W ilcoxon para amostra pareada para cada um dos indicadores dos resultados. E stabeleceu-se nível de significância $\leq 0,05$.

\section{RESULTADOS E DISCUSSÃO}

A seguir são apresentados os resultados da pesquisa, iniciando-se pela caracter ização dos pacientes que fizeram parte da investigação (T abela 1).

T abela 1 - Características dos pacientes incluídos no estudo. Porto Alegre, RS, 2008.

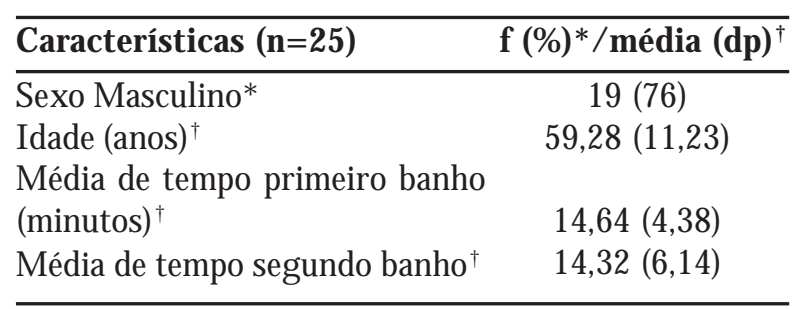

* variável quantitativa; ${ }^{\dagger}$ variável quantitativa.

Quanto à caracterização da amostra, a média de idade dos pacientes de 59,28 anos corrobora a literatura quanto à indicação de Artroplastia Total de Quadril (AT Q) ser preferencial mente para pessoas acima de 60 anos. E mbora as cirurgias de substituição de uma articulação estejam sendo aperfeiçoadas nos últimos anos, com estudos direcionados a abordagens cirúrgicas e novos designs de próteses, o uso subseqüente da articulação implica em desgaste da prótese e na necessidade de substituição(14).

Quanto ao tempo despendido no banho dos pacientes que real izaram AT Q, não houve diferença significativa entre esta atividade no primeiro dia de pós-operatório e 48 horas após. E steachado evidencia que a carga de trabal ho da equipe de enfermagem permanece inalterada neste quesito, apesar da melhora do estado de saúde do paciente.

$\mathrm{Na}$ T abela 2, observa-se que as escalas dos quatro resultados estudados para o DE D éficit no A utocuidado: banho/ higiene apresentaram consistência interna, visto que todas obtiveram alfa de Crombach $=0,6$, demonstrando que as escalas NOC para os quatro resultados estudados são confiáveis para medir a evolução de pacientes com problemas ortopédicos ${ }^{(15)}$.

T abela 2 - Valores do Alfa de Crombach para os Resultados de Enfermagem NOC. Porto Alegre, RS, 2008.

\begin{tabular}{lc}
\hline $\begin{array}{c}\text { Resultados de Enfermagem } \\
\text { NOC (n=4) }\end{array}$ & $\begin{array}{c}\text { Alfa de } \\
\text { Crombach }\end{array}$ \\
\hline Autocuidado: A tividades de Vida & \\
Diária & 0.885 \\
Autocuidado: Banho & 0.899 \\
Autocuidado: H igiene & 0.674 \\
Autocuidado: Higiene Oral & 0.915 \\
\hline
\end{tabular}

D os 24 indicadores para os quatro resultados NOC que constam na T abela 3, em 21 foi possível monitorar a evolução do paciente durante a implementação dos cuidados de enfermagem, visto que houve variação estatisticamente significativa entre as médias de pontuação do primeiro para 0 segundo banho dos pacientes. Este achado sugere que a NOC pode ser uma ferramenta útil para monitorar os resultados dos pacientes com problemas ortopédicos durante a implementação das intervenções de enfer magem refer ente à higiene corporal.

I nternacional mente, estudos têm sido conduzidos para estudar e testar a utilidade da NOC na assistência. Estudo publicado recentemente no Brasil apontou que o crescente interesse na utilização da N OC na prática clínica deve-se à necessidade de avaliar a qualidade do cuidado prestado aos clientes, além de ser uma exigência do sistema de saúde em decorrência dos custos cada vez mais 
el evado. No estudo, que realizou revisão integrativa da produção de conhecimento acerca da N OC, observou-se que $40 \%$ dos trabalhos da amostra con- templaram a utilização da N OC na assistência revelando que esta já vem sendo utilizada na prática assistencial(16).

T abela 3 - Níveis de significância para comparação dos indicadores dos Resultados de Enfermagem N OC entre a primeira e a segunda observação do banho dos pacientes. Porto Alegre, RS, 2008.

\begin{tabular}{|c|c|c|c|}
\hline Indicadores ( $\mathrm{n}^{\circ}$ pacientes) & $\begin{array}{c}\text { Média } \\
\text { indicadores } \\
10 \text { banho }\end{array}$ & $\begin{array}{c}\text { Média } \\
\text { indicadores } \\
\text { 20 banho }\end{array}$ & $\begin{array}{c}\text { Nível de } \\
\text { significância }\end{array}$ \\
\hline \multicolumn{4}{|l|}{ Autocuidado: Atividades de Vida Diária } \\
\hline Alimentação (21) & 3,28 & 4,24 & 0,008 \\
\hline Vestir-se (24) & 1,76 & 2,76 & 0,000 \\
\hline H igiene Íntima (25) & 1,72 & 2,84 & 0,001 \\
\hline Banho (25) & 1,84 & 2,80 & 0,000 \\
\hline Higiene (19) & 2,18 & 3,13 & 0,003 \\
\hline Higiene Oral (21) & 3,40 & 3,80 & 0,009 \\
\hline A mbulação: Andar (25) & 1,12 & 2,08 & 0,000 \\
\hline A mbulação: Cadeira de rodas (24) & 1,00 & 1,80 & 0,001 \\
\hline D esempenho na transferência (25) & 1,44 & 2,36 & 0,000 \\
\hline \multicolumn{4}{|l|}{ Autocuidado: Banho } \\
\hline Entra no banheiro e sai dele (25) & 1,12 & 2,08 & 0,000 \\
\hline Obtém itens para o banho (25) & 1,76 & 2,08 & 0,046 \\
\hline Consegue água (25) & 1,16 & 1,72 & 0,010 \\
\hline Abre a tor neira (25) & 1,20 & 2,00 & 0,024 \\
\hline Regula a temperatura da água (25) & 1,60 & 2,68 & 0,001 \\
\hline Banha-se no chuveiro (25) & 1,16 & 2,44 & 0,000 \\
\hline Lava o corpo (24) & 1,36 & 2,80 & 0,000 \\
\hline Seca o corpo (24) & 1,40 & 2,68 & 0,000 \\
\hline \multicolumn{4}{|l|}{ Autocuidado: Higiene } \\
\hline Lava as mãos (24) & 2,16 & 3,84 & 0,001 \\
\hline Limpa a área do períneo (24) & 1,64 & 2,96 & 0,001 \\
\hline M antém a higiene oral (18) & 3,16 & 3,60 & 0,015 \\
\hline \multicolumn{4}{|l|}{ Autocuidado: Higiene Oral } \\
\hline Escova os dentes (15) & 3,24 & 3,36 & 0,063 \\
\hline Limpa a boca, as gengivas e a língua (16) & 3,44 & 3,32 & 0,102 \\
\hline L impa a dentadura ou aparel hos dentários (6) & 1,12 & 1,40 & 0,317 \\
\hline M anuseia o equipamento necessário (16) & 3,36 & 3,88 & 0,038 \\
\hline
\end{tabular}

A penas três indicadores: E scova os dentes, Limpa a boca, as gengivas e a língua e Limpa a dentadura ou aparel hos dentários, pertencentes ao resultado Autocuidado: H igiene O ral, não apresentaram diferença estatisticamente significativa entre a primeira ea segunda coleta, possivel mente pelo número reduzido de observações. M uitos pacientes recusavam-se a realizar a higiene oral na ocasião do banho, momento em que eram observados pelos coletadores de dados.

Sabe-se que a doença periodontal tem sido associada a diver sas doenças e que o controle de placa tor nou-se uma necessidade de saúde pública, uma vez que a presença de patógenos bucais po- dem interferir negativamente no status de saúde do paciente. As complicações decorrentes da falta ou inadequação do procedimento de higiene oral podem aumentar o tempo de permanência hospitalar em 6,8 a 30 dias, fazendo-se necessário o controle mecânico da placa bacteriana através da escovação e uso de fio dental|(17). É neste contexto que a equipe de enfer magem está inserida, prestando 0 cuidado de acordo com o grau de dependência do paciente. Desta maneira, mesmo os indicadores Escova os dentes, Limpa a boca, as gengivas e a língua e Limpa a dentadura ou aparelhos dentários do resultado de enfermagem Autocuidado: Higiene O ral não tendo apresentado varia- 
ção estatisticamente significativa entre o primeiro e segundo banho, a manutenção da higiene oral é entendida como parte importante da assistência de enfer magem pelo enfermeiro e está descrita na literatura ${ }^{(17)}$.

Encontrou-se dificuldades com a escal a L ikert apresentada pela NOC para os resultados, porque não possibilitava mensurar adequadamente todos os indicadores. Por exemplo, na pontuação do indicador Regula a temperatura da água o escore 3 da escala Likert era definido como Precisa de mecanismo auxiliar, não adequando-se ao indicador. U tilizou-se a segunda edição da N OC para a coleta de dados, por ser a única traduzida para a língua portuguesa do Brasil no momento da construção do instrumento de col eta de dados. $N$ a quarta edição os ter mos usados como âncor as na escala Likert foram modificados para 1 = extremamente comprometido a 5 = não comprometido, corroborando as limitações encontradas nesta pesquisa ${ }^{(5)}$.

Pesquisadores da City U niversity of $\mathrm{N}$ ew $\mathrm{Y}$ ork publicaram estudo de caso utilizando o resultado de enfermagem Autocuidado: Atividades de Vida D iária para paciente idosa com artrite não hospitalizada e consider aram aplicáveis a maioria dos indicadores. Os resultados foram discutidos em dois momentos: primeiramente foi realizada uma avaliação da paciente e pontuados os indicadores. A pós, foram el eitas pontuações como metas a serem al cançadas com a implementação do plano de cuidados e mensurada na avaliação subsequente ${ }^{(18)}$. N este estudo foi utilizada a quarta edição da N OC. D este modo, os ter mos âncoras da escala estavam atual izados não sendo evidenciadas as dificuldades que encontrou-se no presente estudo.

E m estudo piloto conduzido em um hospital universitário brasileiro e recentemente publicado, as autor as real izaram a val idação das principais intervenções da NIC e dos resultados sugeridos na NOC para o diagnóstico de enfermagem Volume de Líquidos Excessivo. Sete enfermeiros especialistas em cardiologia realizaram a validação através do preenchimento de um instrumento construído para o estudo, contendo uma escala Likert de cinco pontos. D os 53 indicadores contidos nos resultados de enfermagem $N O C$ sugeridos para 0 referido diagnóstico apenas 8 obtiveram escore inferior a 0.5 , sendo descartados. A presentaram escore superior a 0.8 , sendo considerados maiores, 26 indicadores. Este estudo demonstra a necessidade de serem validados os resultados e seus indi- cadores na prática clínica brasileira, uma vez que as autoras mencionam não existir outros estudos semel hantes no Brasil que possibilitem comparar seus resultados ${ }^{(19)}$.

De modo semel hante, o grande número de indicadores utilizados na presente investigação e que foram validados em etapa anterior por enfermeiras peritas, apontam para a necessidade de desenvolver estudos de validação clinica a fim de que sejam criadas condições favoráveis à implantação e utilização das taxonomias na prática.

E $m$ outra investigação, realizada na Califórnia, foi proposto e introduzido terminologias padronizadas de enfermagem para um Sistema de Resposta Rápida, que consiste no atendimento de situações de agravo do paciente e é liderado por enfermeiros. Este estudo utilizou como fonte de dados além da experiência clínica pessoal, literatura atual, estudos de investigação e meta-análise. Os resultados da investigação apontaram que os atuais diagnósticos, as intervenções e os resultados de enfermagem listados, respectivamente, na NANDA-I, NIC e NOC são imprecisos ou inadequados para descrever o cuidado de enfermagem durante uma situação de risco de vida. Consideram que o uso de terminologia padronizada de enfermagem é favorável ao contatar um médico para transmitir informações sobre um incidente crítico, tais como hipotensão profunda, a fim de prevenir uma parada cardiorrespir atória. N ão ter ou não usar a terminologia padronizada pode atrasar decisões ou criar confusão levando a decisão errada. 0 estudo mostrou que a utilização das ligações das classificações melhora 0 atendimento prestado ao paciente oferecendo recursos adicionais e um nível de conhecimento maior que será transmitido ao colega do turno posterior para continuar a prestar assistência. T ambém foi verificada a diminuição de custos quando o agravo do paciente é evitado. E m suma, os resultados da investigação mostraram que a utilização das terminologias de enfermagem faz a diferença na qualidade ou na duração da vida de um paciente(20).

\section{CONCLUSÕES}

0 estudo possibilitou verificar a aplicabilidade dos quatro resultados da Classificação NOC (Autocuidado: A tividades de Vida D iária, A utocuidado: Banho, Autocuidado: Higiene, A utocuidado: H igiene Oral) em pacientes submetidos à AT Q com 
D éficit no Autocuidado: banho/ higiene. M esmo possibilitando a monitorização da evolução dos pacientes, identificou-se inadequações dos termos utilizados como âncoras na escala Likert, que foram ratificadas pelos pesquisadores da classificação, ao modificarem esses itens na última edição da NOC.

Observou-se a dificuldade de utilizar 24 indicadores para mensurar a independência do paciente. Assim, como sugerido pelas pesquisadoras da NOC, concluiu-se que o ideal seria aplicar poucos e significativos indicadores na prática clínica.

Sugere-se que sejam desenvolvidos estudos semel hantes em outras instituições para que metanálises possam ser realizadas quando as variáveis forem similares.

Independente de qual DE o paciente estiver apresentando, as intervenções precisam atender suas necessidades. $\mathrm{E}$ o resultado obtido com as intervenções pode ser mensurado com a utilização da NOC, por meio de seus indicadores e escalas. $\mathrm{N}$ este sentido, torna-se imperativo empenhar mos esforços no desenvolvimento e validação de mais esta classificação que vem auxiliar a enfermagem na prática clínica, no ensino e na pesquisa.

\section{REFERÊNCIAS}

1 T homéE S, Vieira DS, Silva PF, Johansson SC, Sottomaior VS, D ias VL M , et al., organizadores. Sistematização da assistência de enfermagem no HCPA. Porto Alegre: HCPA; 2006.

2 Crossetti M GO, Rodegheri M , D 'Á vila M L, Dias VLM . 0 uso do computador como ferramenta para implantação do Processo de Enfermagem. Rev Bras Enferm. 2002;55(6):705-8.

3 Benedet SA, Bub M BC. M anual de diagnóstico de enfermagem: uma abordagem baseada na teoria das necessidades humanas e na classificação diagnóstica da NAN DA. 2ª ed. F lorianópolis: Bernúncia; 2001.

4 D ochterman JC, Bulechek G M. Classificação das Intervenções das E nfermagem (NIC). 4a ed. Porto Alegre: Artmed; 2008.

5 M oorhead S, Johnson M, M aas M, Swanson E. N ursing Outcomes Classification (NOC). $4^{\text {th }}$ ed. Philadelphia: Elsevier; 2008.

6 Johnson M, M aas M, M oorhead S. Classificação dos Resultados de E nfermagem (N OC). $2^{a}$ ed. Porto Alegre: Artmed; 2004.
7 Almeida M A, Cezaro P, Longaray VK. Diagnósticos de enfer magem preval entes e cuidados prescritos para pacientes ortopédicos. Online Braz J N urs [ I nternet] . 2006 [ citado 2009 nov 22] ;5(3). D isponível em: http:/ / www.objnursing.uff.br/index.php/ nursing/ article/ view/502/ 115.

$8 \mathrm{~N}$ or th A merican N ursing Diagnosis A ssociation - Inter national. Diagnósticos de enfermagem da NAN DA: definições e classificação 2009-2011. Porto Alegre: Artmed; 2010.

9 Johnson $M$, Bulechek $G$, D ochterman J M, M aas M, M oorhead S. NANDA, NOC and NIC Linkages. $2^{\text {nd }}$ ed. St. Louis: M osby; 2006.

10 M acedo CAS, G alia CR, Rosito R, Perea CE F, M üller $L M$, Verzoni $G G$, et al. A bordagem cirúrgica na artroplastia total primária de quadril: ântero-lateral ou posterior? Rev Bras Ortop [ Internet] . 2002 [ citado 2009 nov 15];37(9):387-91. Disponível em: http:/ / www.rbo.org.br/ pdf/ 2002_set_08.pdf.

11 Almeida M A, L ongaray VK, Cezaro P, Barilli SL S. Correspondência entre cuidados prescritos para pacientes com probl emas ortopédicos a classificação das intervenções de enfermagem. Rev G aúcha Enferm. 2007;28(4):480-8.

$12 \mathrm{G}$ oldin J R. M anual de iniciação à pesquisa em saúde. $2 ª$ ed. Porto Alegre: D acasa; 2000.

13 M inistério da Saúde (BR ), Consel ho N acional de Saúde. Resolução no 196, de 10 de outubro de 1996: dispõe sobre as diretrizes e as nor mas regulamentadoras de pesquisa envolvendo seres humanos. Brasília (DF); 1996.

14 Kneale J, D avis P. Orthopaedic and trauma nursing. $2^{\text {nd }}$ ed. London: Churchill Livinstone; 2005.

15 Polit DF, Beck CT, H ungler BP. F undamentos de pesquisa em enfermagem: métodos, aval iação e utilização. 5a ed. Porto Alegre: Artmed; 2004.

$16 \mathrm{G}$ arbin LM , Rodrigues CC, Rossi LA, Carvalho EC. Classificação de resultados de enfermagem (NOC): identificação da produção científica relacionada. Rev G aúcha Enferm. 2009;30(3):508-15.

17 M artins G C, Santos N O, G omes ET. Higiene or al: atuação da equipe de enfer magem em paciente com déficit no autocuidado. Rev E nferm I ntegrada. 2009; 2(1):144-51.

18 Cirminiello C, T erjesen M . Casestudy home nursing care for a 62-year-old woman with multiple health 
problems. Int J Nurs Terminol Classif. 2009;20(2): 96-9.

19 Lopes JL, Barros ALBL, M ichel JL M . A pilot study to validate the priority nursing interventions classification interventions and nursing outcomes classification outcomes for the nursing diagnosis "excess fluid volume" in cardiac patients. Int J N urs Terminol Classif. 2009;20(2):76-88.
20 Wong E. N ovel nursing terminologies for the Rapid ResponseSystem. Int J N urs Terminol Classif. 2009; 20(2):53-63.

\section{AGRADECIMENT OS}

Ao $\mathrm{F}$ undo de Incentivo à Pesquisa do $\mathrm{H}$ ospital de Clínicas de Porto A legre (FIPE / HCPA) pelo apoio financeiro.
Endereço da autora / Dirección del autor / Author's address:

M iriam de Abreu Almeida

Rua São M anoel, 963, Rio Branco

90620-110, Porto Alegre, RS

E-mail: miriam.abreu@terra.com.br
Recebido em: 01/ 02/ 2010

A provado em: 15/ 03/ 2010 